\title{
Polarization of ferroelectric films through electrolyte
}

\author{
Henrik Toss, Negar Sani, Simone Fabiano, Daniel T Simon, \\ Robert Forchheimer and Magnus Berggren
}

\section{Linköping University Post Print}

\section{Tweet}

N.B.: When citing this work, cite the original article.

Original Publication:

Henrik Toss, Negar Sani, Simone Fabiano, Daniel T Simon, Robert Forchheimer and Magnus Berggren, Polarization of ferroelectric films through electrolyte, 2016, Journal of Physics, (28), 10.

http://dx.doi.org/10.1088/0953-8984/28/10/105901

Copyright: IOP Publishing: Hybrid Open Access

http://www.iop.org/

Postprint available at: Linköping University Electronic Press

http://urn.kb.se/resolve?urn=urn:nbn:se:liu:diva-121802 


\title{
Polarization of ferroelectric films through electrolyte
}

Henrik Toss ${ }^{1}$, Negar Sani ${ }^{1}$, Simone Fabiano ${ }^{1}$, Daniel T Simon ${ }^{1}$, Robert Forchheimer ${ }^{2}$, Magnus Berggren $^{1 *}$

1. Laboratory of Organic Electronics, Department of Science and Technology, Linköping University, SE-601 74 Norrköping, Sweden.

2. Division of Information Coding, Department of Electrical Engineering, Linköping University, SE- 581 83 Linköping, Sweden

* Corresponding author. Email: magnus.berggren@liu.se

\begin{abstract}
A simplified model is developed to understand the field and potential distribution through devices based on a ferroelectric film in direct contact with an electrolyte. Devices based on the ferroelectric polymer polyvinylidenefluoride-trifluoroethylene (PVDF-TrFE) were produced - in metalferroelectric-metal, metal-ferroelectric-dielectric-metal, and metal-ferroelectric-electrolyte-metal architectures - and used to test the model, and simulations based on the model and these fabricated devices were performed. From these simulations we find indication of progressive polarization of the films. Furthermore, the model implies that there is a relation between the separation of charge within the devices and the observed open circuit voltage. This relation is confirmed experimentally. The ability to polarize ferroelectric polymer films through aqueous electrolytes, combined with the strong correlation between the properties of the electrolyte double layer and the device potential, opens the door to a variety of new applications for ferroelectric technologies, e.g., regulation of cell culture growth and release, steering molecular self-assembly, or other large area applications requiring aqueous environments.
\end{abstract}

\section{INTRODUCTION}

The vinylidene fluoride monomer is commonly incorporated to provide the permanent electrical polarization in different ferroelectric polymer systems, such as in polyvinylidenefluoridetrifluoroethylene (PVDF-TrFE). While directly sandwiched between two electrodes, the piezo-, pyroand ferroelectric properties of such polymers have extensively been explored and utilized in various sensor, transducer, memory and actuator devices [1-6]. Recently, ferroelectric polymers have also been shown to be able to stabilize electric double layers (EDL) in polyelectrolyte thin films. This was used to produce low voltage-operating transistor memory devices [7]. In a wider sense, the possibility of polarizing a ferroelectric film through an electrolyte, and thus controlling the charge and dipole characteristics along the ferroelectric/electrolyte (FE) interface, can potentially open up for many new opportunities for electrolyte-based or electrochemical devices in electronics and bioelectronics applications. To aid this development it is critical to establish a model of such device systems that include the statics and dynamics of charge polarization, electric fields and potentials that are generated upon biasing the device. Proper understanding of the mechanisms behind the operation of the FE interface will enable engineers and scientists to predict the behavior of novel ferroelectric-electrolyte-based devices. Much theoretical work has previously been devoted to describe and understand the mechanisms in ferroelectric switching materials when sandwiched between metallic or semiconductor materials [8-13]. Here, we report a simplified model of a 
capacitor structure in which an electrolyte has been included between a first bare metal electrode and the ferroelectric material in contact with a second metal electrode (Fig. 1). We also present experimental data from such a device, based on an aqueous electrolyte and a PVDF-TrFE ferroelectric polymer, and compare its output electrical characteristics with the expected behavior predicted by our model. Furthermore we see that the model should expand also to simple dielectrics in place of the electrolyte and such devices are also produced and measured on for control. The suggested model, here proposed, leads us to conclude that there are limiting factors, in these kinds of device configurations, which must be considered before developing new ferroelectric-electrolyte-based devices. In particular, we find that the ferroelectric film thickness and the properties of the stabilized/stabilizing electric double layer in the electrolyte must be considered in order to obtain a proper operating device system. Furthermore we note that there is a measurable voltage produced over the polarized devices related to the separation of charge between the different layers of the devices.

a)

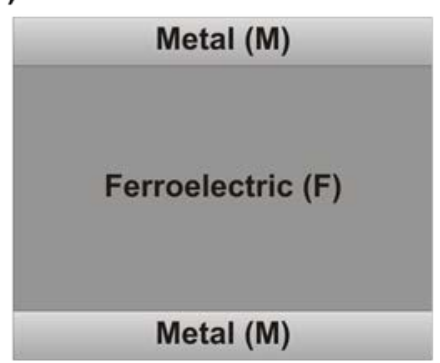

b)

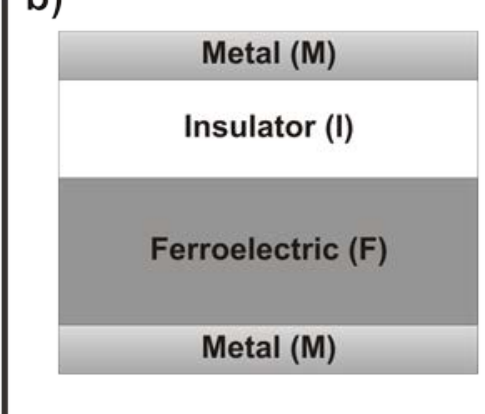

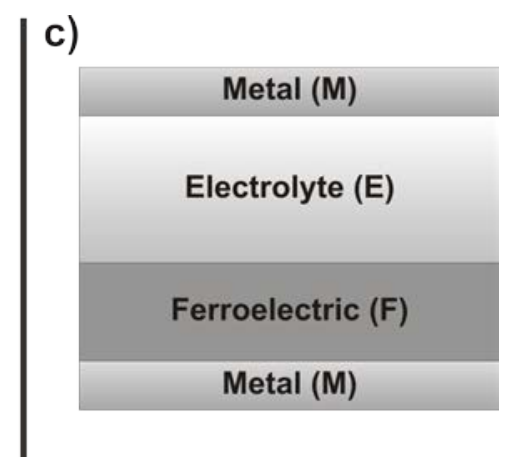

FIG 1. Schematic image of the device architecture for with the materials stacked from bottom to top in accordance with the name of the device in question; MFM (a), MFIM (b) and MFEM (c). The proportions of the different layers are meant only as representations and are not according to scale with any of the experimental devices.

\section{EXPERIMENTAL}

\section{A. Fabrication}

Ti bottom electrodes were deposited on SiOx substrates through thermal evaporation. Films of the ferroelectric polymer PVDF-TrFE, with a monomer weight ratio of 70:30, were prepared on top of the Ti bottom electrodes by spin coating PVDF-TrFE from a Methyl-Ethyl-Ketone (MEK) solution. The samples were then annealed at a temperature of $134{ }^{\circ} \mathrm{C}$ for $30 \mathrm{~min}$. The resulting thickness of the ferroelectric film was estimated to be $\sim 1.2 \mu \mathrm{m}$, which we found from measurements performed on parallel samples using a Dektak profilometer.

To form the metal/ferroelectric/electrolyte/metal structure (MFEM), a drop of Phosphate Buffered Saline (PBS) was placed on the film. An Al wire was then dipped into the droplet and used as the top electrode for the MFEM.

When preparing the metal/ferroelectric/insulator/metal structure (MFIM) a thin ( 20-30 nm) insulating layer of PolyMethylmethacrylate (PMMA) was spin coated on top of the PVDF-TrFE from solution in DiEthyl Carbonate (DEC). For the metal/ferroelectric/metal (MFM) and MFIM devices Ti was evaporated onto the samples to serve as top electrodes.

\section{B. Measurements}


A Current-Voltage sweep (I-V) was performed on the samples using a Keithley 4200-SCS parameter analyzer. The voltage sweeps were performed by elevating the voltage from $0 \mathrm{~V}$ to $90 \mathrm{~V}$ and then reversed until $V<-90 \mathrm{~V}$ to finally be reversed another time until $\mathrm{V}=0 \mathrm{~V}$ again. As a peak voltage of 90 $\mathrm{V}$ did not seem to completely switch the MFIM devices, a peak voltage value of $180 \mathrm{~V}$ was used instead to guarantee that the devices were completely polarized.

Following the polarization of the devices, a zero current condition was set after which the resulting voltage was acquired. We refer to this voltage as the Open Circuit Voltage $\left(\Phi_{\text {oc }}\right)$.

III. RESULTS AND DISCUSSION

C. Current-Voltage measurements
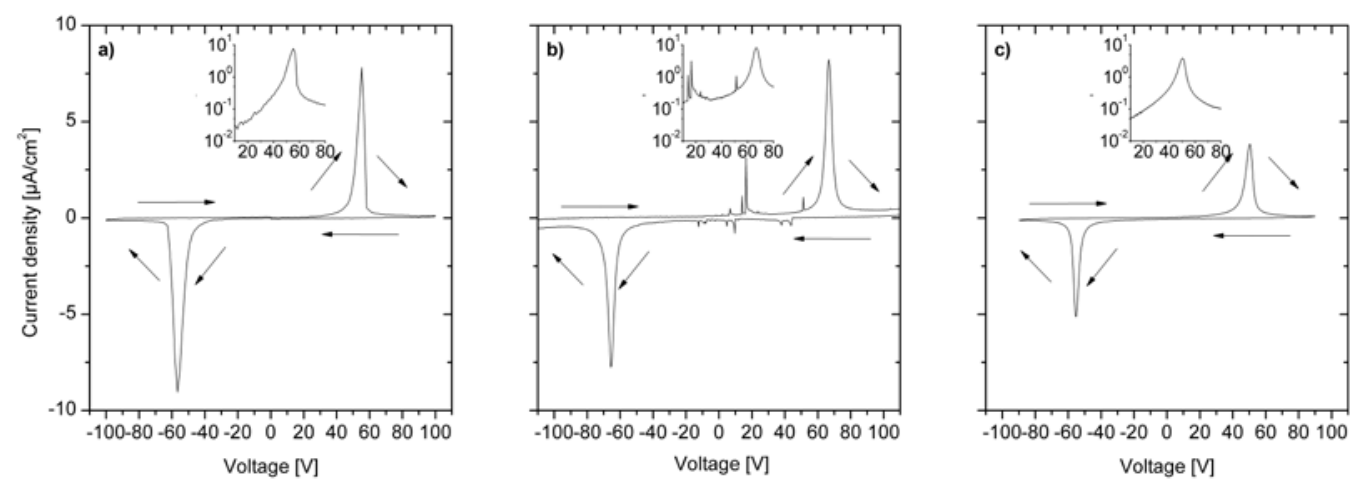

FIG 2. Current-Voltage (I-V) characteristics showing the ferroelectric response of the different devices, MFM (a), MFIM (b) and MFEM (c). The arrows indicate sweep direction. In the insets the current peaks of the polarization curves for positive voltages can be seen with the current on a log-scale.

The curves from the I-V sweeps of the three devices are given in Fig. 2. In all the three (MFM, MFIM and MFEM) cases clear peaks are found, which corresponds to the ferroelectric polarization switching. It is hard to mark a definite beginning and end of the polarization peaks, but as can be seen in the insets of Fig. 2 the increase in current, attributed to ferroelectric polarization, appears to begin at lower voltages for the MFIM, as compared to MFM and MFEM, indicative of a lower onset voltage for the ferroelectric polarization in the MFIM. The remnant surface polarization charge $\left(P_{s}\right)$ was extracted through integration of the current along the sweep for each voltage flank. The voltage that corresponds to the current peak $\left(U_{\text {peak }}\right)$, occurring as the ferroelectric dipoles switches was also extracted. This value was also used to calculate an estimation of the Coercive Field $\left(E_{c}\right)$, i.e. the field inside the ferroelectric thin film needed to switch the ferroelectric polarization. A summary of the extracted results is provided in table I together with the measured $\Phi_{o c}$ for all three devices.

\section{Theoretical Model and Reasoning}

A model was constructed to map the voltage distribution throughout the three different ferroelectric device systems. For simplicity reasons and for easy comparisons to measured results, we have simplified the system by always assuming static conditions. This means that the field inside the electrolyte $\left(E_{E}\right)$ and the metal $\left(E_{M}\right)$ electrodes is always zero. Furthermore we assume that the charge at the interfaces is to be uniformly distributed along the surfaces, with zero extension perpendicular 
to the surface interfaces (Fig. 3). This provides a 1D system similar to what has previously been used to describe both ferroelectric[8] and electrolyte[14] capacitor systems separately.

The resulting net surface charge density generated and established by the ferroelectric polarization $\left(P_{s}\right)$ is non-Faradic and is assumed to be located along the very surface of the ferroelectric film and not inside the ferroelectric material. The mobile charges in the different layers are assumed to be separated from the adjacent layer by material-specific lengths. These lengths could for instance correspond to the screening length, in the electrodes $\left(t_{e}\right)$, and to the electric double layer thickness, in the electrolyte $\left(t_{H h}\right)$. For this reason we here use the denotation $E_{e}$ for the electric field at the electrode interfaces and $E_{\mathrm{Hh}}$ for the electric field at the electrolyte interfaces. From Kirchoff's laws we know that the mobile charge density (q) at the interfaces, throughout the systems must be equal in magnitude. Based on these assumptions it is now possible to derive the equations for the electric field and potentials throughout the system as functions of mobile charge density. Inside the ferroelectric film and insulating films the mobile charge density is constant and zero and the field is thus constant throughout these films ( $E_{F}$ and $E_{1}$ respectively). With subscripts denoting the layers; bulk of the metal electrodes (M), metal electrodes at the surfaces/interfaces (e), inside the ferroelectric $(F)$, inside the dielectric (I), in the EDL (Hh) and bulk of the electrolyte (E), The field, $E$, can be written as

$$
\begin{aligned}
& E_{M}=0, \\
& E_{e}=\frac{q}{\varepsilon_{e}}, \\
& E_{F}=\frac{q-P_{s}}{\varepsilon_{F}}, \\
& E_{I}=\frac{q}{\varepsilon_{I}}, \\
& E_{H h}=\frac{q}{\varepsilon_{H h}}, \\
& E_{E}=0,
\end{aligned}
$$

where $\varepsilon$ denotes the dielectric constants of the different layers, with denotations corresponding to each layer, respectively.

As the field $\left(E_{i}\right)$ in the different sections of the system is approximated as constant throughout their corresponding layers (or layer interfaces) the potential increase throughout each layer is therefore constant and the total potential, $\Phi$, of the system can be written as the sum:

$$
\Phi=\int \varphi=\sum_{i} t_{i} * E_{i},
$$

where $t_{i}$ denotes the thicknesses of layers and interfaces. For the three types of devices discussed in this work the corresponding potentials would be: 


$$
\begin{aligned}
\Phi_{M F M} & =-2 t_{e} * E_{e}-t_{F}^{*} E_{F} \\
& =-2 t_{e} \frac{q}{\varepsilon_{e}}-t_{F} \frac{q-P_{s}}{\varepsilon_{F}}, \\
\Phi_{M F I M} & =-2 t_{e} * E_{e}-t_{F}^{*} E_{F}-t_{I} * E_{I} \\
& =-2 t_{e} \frac{q}{\varepsilon_{e}}-t_{F} \frac{q-P_{S}}{\varepsilon_{F}}-t_{I} \frac{q}{\varepsilon_{I}}, \\
\Phi_{M F E M} & =-2 t_{e} * E_{e}-t_{F} * E_{F}-2 t_{H h} * E_{H h} \\
& =-2 t_{e} \frac{q}{\varepsilon_{e}}-t_{F} \frac{q-P_{S}}{\varepsilon_{F}}-2 t_{H h} \frac{q}{\varepsilon_{H h}} .
\end{aligned}
$$

a)

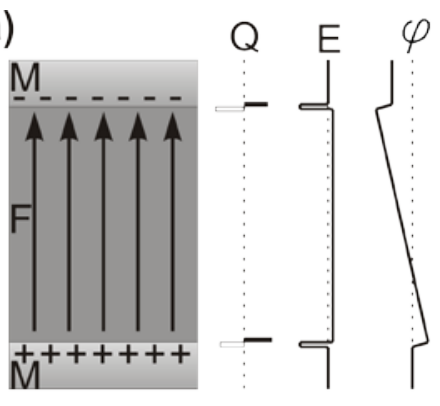

b)

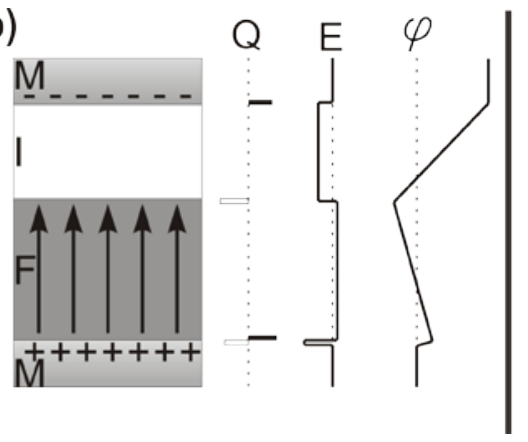

c)

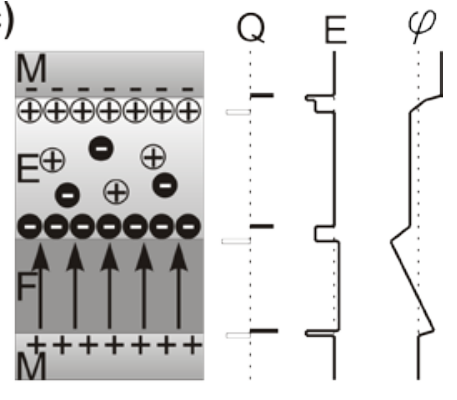

FIG 3. Schematic images of the local net charge density, $Q$, field, $E$, and local electric potential, $\varphi$, throughout the three different devices. MFM (a), MFIM (b) and MFEM (c). Note that the values in the schematic graphs are just examples of possible charge distributions and that there is no relation between the size of the dipoles and the arrows representing the direction of the polarization of the films. In the MFEM the ions of the electrolyte are drawn as organizing into a dense Helmholtz plane.

When the polarized film is placed in direct contact with a metal electrode the charge in the electrode will rearrange to compensate for the immobile surface charge of the ferroelectric layer. When the system has been stabilized the mobile charge density of the electrode will equal the immobile surface polarization charge density of the polarized ferroelectric layer. Applying these conditions to the equations in the proposed model suggests measuring $\Phi_{\mathrm{Oc}}$ of the polarized systems under a zerocurrent condition. We then get:

$$
\begin{aligned}
& \Phi_{O C, M F M}=-2 t_{e} \frac{P_{s}}{\varepsilon_{e}}, \\
& \Phi_{O C, M F I M}=-2 t_{e} \frac{P_{s}}{\varepsilon_{e}}-t_{I} \frac{P_{s}}{\varepsilon_{I}}, \\
& \Phi_{O C, M F E M}=-2 t_{e} \frac{P_{s}}{\varepsilon_{e}}-2 t_{H h} \frac{P_{s}}{\varepsilon_{H h}} .
\end{aligned}
$$

From literature we find estimates for the dielectric constants of included layers. Typically, $\varepsilon_{\mathrm{e}} \approx \varepsilon_{0}$ at metal surfaces and $\varepsilon_{\mathrm{Hh}} \approx 6^{*} \varepsilon_{0}$ in salt water [15]. Using an estimated relative permittivity of 4.9 for PMMA [16] it is possible to calculate the thicknesses of critical interfaces and layers separating 
charges, from the experimentally achieved $\Phi_{\text {oc }}$. By first extracting $t_{e}$ from the MFM results and using that value as an estimate for $t_{e}$ in the other two devices we achieve (table I):

TABLE I. Experimentally extracted parameters and thicknesses calculated from these using the suggested model.

\begin{tabular}{ccccccc}
\hline \hline $\begin{array}{c}\text { Device } \\
\text { structure }\end{array}$ & $\begin{array}{c}\text { Extracted } \mathrm{P}_{\mathrm{s}} \\
(\mathrm{mC} / \mathrm{m} 2)\end{array}$ & $\begin{array}{c}\Phi_{\mathrm{OC}} \\
(\mathrm{V})\end{array}$ & $\begin{array}{c}\mathrm{U}_{\text {peak }} \\
(\mathrm{V})\end{array}$ & $\begin{array}{c}\mathrm{t}_{\mathrm{F}} \\
(\mu \mathrm{m})\end{array}$ & $\begin{array}{c}\text { Extracted } \mathrm{E}_{\mathrm{C}} \\
(\mathrm{MV} / \mathrm{m})\end{array}$ & $\begin{array}{c}\text { Resulting calculated thicknesses } \\
(\AA)\end{array}$ \\
\hline $\mathrm{MFM}$ & 100 & 0.5 & 57 & 1.2 & 48 & $\mathrm{t}_{\mathrm{e}}=0.22$ \\
MFIM & 40 & 24 & 65 & 1.2 & 52 & $\mathrm{t}_{\mathrm{l}}=258$ \\
MFEM & 60 & 1.6 & 55 & 1.2 & 46 & $\mathrm{t}_{\mathrm{Hh}}=5.8$ \\
\hline \hline
\end{tabular}

We note that the thicknesses calculated from the experimental data, using our model, correspond well to actual physical parameters, found in literature. The calculated $t_{e}=0.22 \AA$ is rather close to expected screening lengths of a metal. In the case of MFIM the calculated thickness, $t_{1}=25.8 \mathrm{~nm}$, of the PMMA film corresponds very well to the 20-30 nm thickness measured using the profilometer. Regarding the thickness separating the charges in the electrolyte from the adjacent layers, $t_{\mathrm{Hh}}=$ $5.8 \AA$, seems to be in good agreement with expected separation of the charges of the inner Helmholtz plane in an electric double layer, which is given by the ionic radius.

We note that the value of $\Phi_{\text {oc }}$ for the MFEM devices is strongly dependant on the distance of the closest approach for the charges in the electrolyte to the surface of the ferroelectric and top electrode. This strong dependence can be utilized to monitor any dynamic processes present, which may impact the thickness and/or permittivity of the Helmholtz layer, such as a process including an exchange of ionic species or binding of molecules to the surface). Recording $\Phi_{\mathrm{oc}}$ over time may thus provide us with a unique opportunity to study the intimate dynamics of EDLs.

\section{E. Simulations}

Our simple model was used to investigate how the field inside the ferroelectric layer $\left(E_{F}\right)$ and the mobile surface charge (q) along interfaces depends on the applied voltage $\Phi$ for the MFM, MFIM and MFEM configurations. The plots of $q$ and $E_{F}$ versus $\Phi$ obtained from simulations based on the model are given in Fig. 4 and Fig. 5. For these simulations we have included the actual dimensions and physical properties of the different layers. The thickness of the ferroelectric film is set to be $1.2 \mu \mathrm{m}$. From literature we find that the relative permittivity $(\varepsilon)_{\mathrm{F}}$ is $\sim 8$, the ferroelectric coercive field $\left(\mathrm{E}_{\mathrm{C}}\right)$ is $\sim 50 \mathrm{MV} / \mathrm{m}$ and that the remnant polarization $\left(P_{s}\right)$ is $\sim 100 \mathrm{mC} / \mathrm{m}^{2}$ for $\operatorname{PVDF}(70)-\operatorname{TrFE}(30)$ [17-18]. Regarding the FE and EM interfaces we assume that the Helmholtz layer thickness can be approximated by a standard metal/salt water interface, i.e. $t_{\mathrm{Hh}} \sim 1 \mathrm{~nm}$, and that the relative permittivity of salt water inside the Helmholtz layer $\left(\varepsilon_{\mathrm{Hh}}\right)$ is $\sim 6$ [15]. Furthermore, we set the thickness of the insulating layer to $20 \mathrm{~nm}$ with a relative permittivity of $\varepsilon_{1} \sim 4.9$ corresponding to the PMMA used in our actual experiments [16]. 
a)

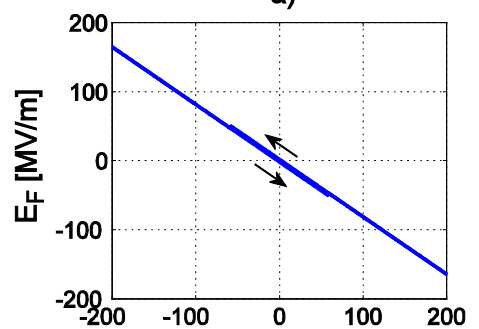

d)

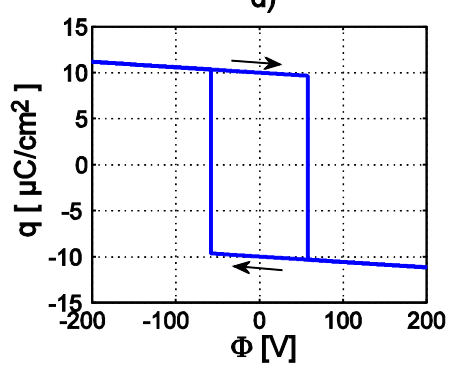

b)

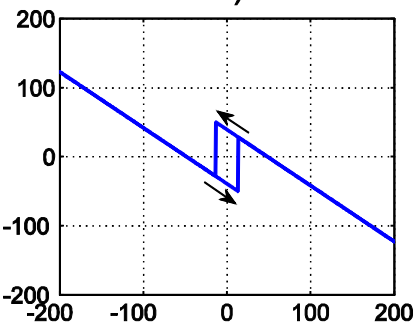

e)

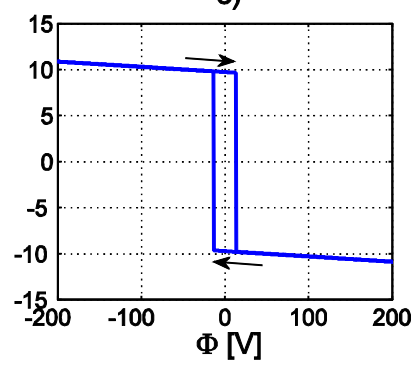

c)

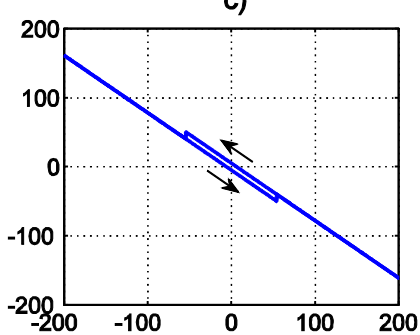

f)

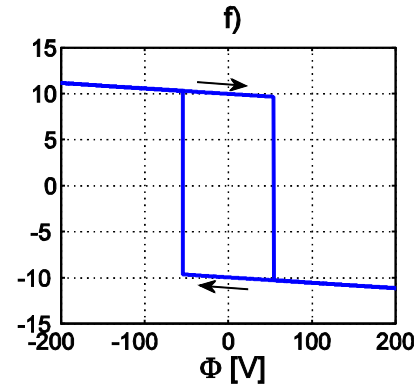

FIG 4. Simulations assuming simultaneous switching of all ferroelectric dipoles. Electric field inside the ferroelectric polymer, EF, and electrode surface charge density, $q$, as function of applied voltage, $\Phi$ MFM (a,d), MFIM (b,e) and MFEM $(c, f)$. The simulations are based on the model using the parameters found in table $I$.

a)

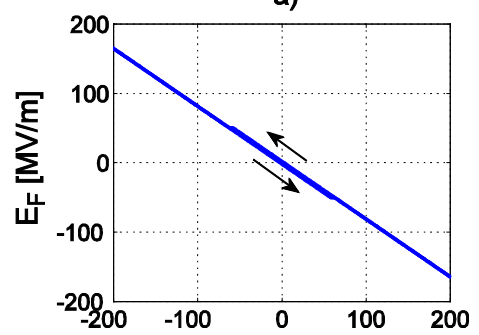

d)

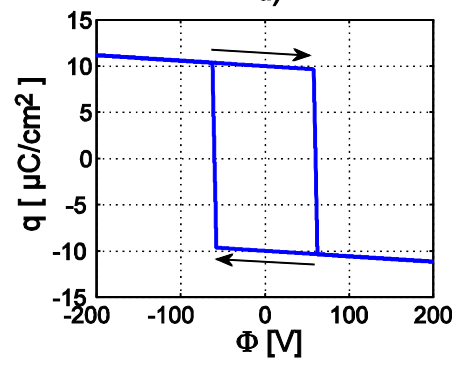

b)

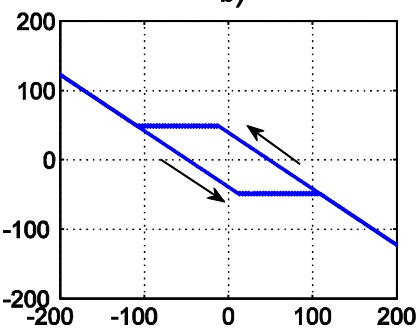

e)

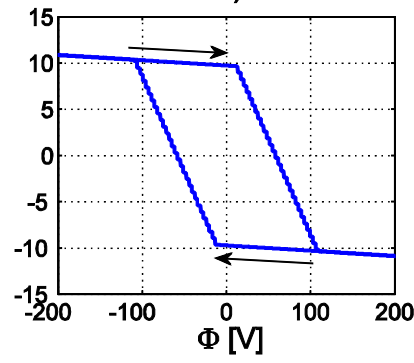

c)

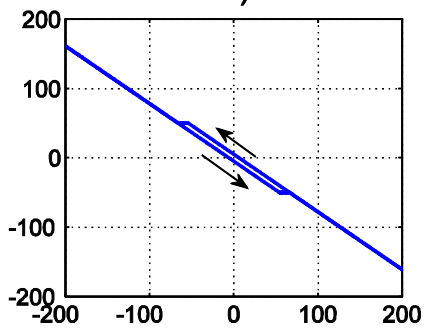

f)

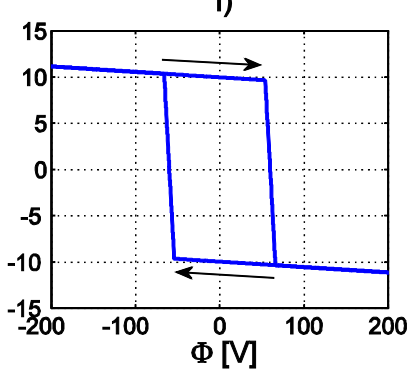

FIG 5. Simulations assuming progressive switching of ferroelectric dipoles. Electric field inside the ferroelectric polymer, $E F$, and electrode surface charge density, $q$, as function of applied voltage, $\Phi$ MFM (a,d), MFIM (b,e) and MFEM (c,f). The simulations are based on the model using the parameters found in table $I$. In these simulations it is assumed that the ferroelectric polarization proceeds successively as the field inside the ferroelectric reaches the coercieve field EC. This can be observed as a plateau in EF as it reaches EC which ends when the film is completely polarized and thus no longer can compensate by rearranging the dipoles further.

When simulating and modeling the reorganization of dipoles within the ferroelectric layer, while its internal field $\left(E_{F}\right)$ approaches the coercive field $\left(E_{C}\right)$, one can either assume that the entire film polarizes instantly or that dipoles successively flip to just barely compensate for the externally 
applied electric field. The differences in $E_{F}$ and electrode surface charge density (q) between these two approaches are found in Fig. 4 (simultaneous polarization) and Fig. 5 (progressive polarization). Assuming simultaneous polarization (Fig. 4) the model indicates that adding a thicker interfacial layer (electrolyte or dielectric) actually lowers the onset voltage of ferroelectric polarization. This result from our modeling is in agreement with what also is found from measurement performed on very thin ferroelectric films. In that case the electrode screening length is attributed to have a larger effect on the electric potential distribution as the ferroelectric film gets thinner [8]. From this we can thus conclude that the relationship between the thickness of the ferroelectric film and any included insulating layers, and not only the thickness of the ferroelectric layer alone, is a crucial design parameter in dictating the driving voltage for ferroelectric thin film devices. The simultaneous polarization model also implies that the peak switching voltage coincides with the onset voltage. However, in Fig. 2 we observe broadened peaks indicative of progressive polarization. Taking this into account we proceeded with adding progressive charge compensation to the model.

The fundamental difference between the MFM, MFIM, and MFEM systems is the separation of charges throughout the systems, which is dominated by the screening length in the metal (MFM), the dielectric thickness (MFIM) or the inner Helmholtz layer thickness (MFEM), cf table I. In the I-V sweeps the current peak is located at approximately $60 \mathrm{~V}$ for the MFM, MFIM, and MFEM cases. However we observe relatively lower onset voltage for the MFIM device, which is also supported by modeling (Fig. 5e). However, the MFEM characteristics are almost identical to that of MFM device with respect to both peak position and width. This similarity holds as long as the ferroelectric film is much thicker than the screening length within the metal or the inner Helmholtz layer thickness. Note that it is the thickness of the inner Helmholtz layer of the electrolyte, and not its overall thickness. Thus, one can define devices with arbitrary electrolyte thickness without increasing polarization voltage. This then opens up for electronic surface switch devices with an active electrode exposed to aqueous electrolytes, e.g. to regulate the growth and release cell cultures [19-20], steer self assembly [21], or other large area applications.

The $\Phi_{\mathrm{oc}}$ is the most significant measured difference in the MFM ( $\left.\Phi_{\mathrm{oc}}=0.5 \mathrm{~V}\right)$, MFIM $\left(\Phi_{\mathrm{oc}}=24 \mathrm{~V}\right)$, and MFEM ( $\Phi_{\mathrm{OC}}=1.6 \mathrm{~V}$ ) characteristics. Using the model and measured $\Phi_{\mathrm{OC}}$ it is then possible to calculate the charge separation lengths for each structure. The dominating charge separation in the MFM, $t_{e}$, is calculated to be $0.22 \AA$ which corresponds very well to the screening length of a metal. For the MFIM device the calculated dominating charge separation, $t_{1}$, is $258 \AA$, again well in accordance with the thickness of the corresponding layer, i.e. the dielectric. In the case of the MFEM devices the dominating charge separation is in the Helmholtz layers at the FE and EM interfaces. Assuming they are more or less similar the charge separation length within them, $t_{H h}$, is calculated to be $5.8 \AA$, corresponding very well to the thickness of the inner Helmholtz layer, i.e. approximately the radius of the ions in the electrolyte. Our results indicate the possibility to detect thicknesses of layers down to, and even beyond, atomic dimensions, in the case of the MFM devices. In the more complex MFEM and MFIM devices some precision is likely lost, as there are more interfaces in play, but we are still able to measure with precision within the radius of the ions of the electrolyte solution. The dielectric properties of the EDL may differ depending on the electrolyte used. By simply exchanging the electrolyte, while keeping the ionic composition the open circuit voltage might also provide an easy way to acquire the dielectric properties of the EDL in electrolytes, which may differ from the bulk of the electrolyte. As the size of the ionic species of the electrolyte appears to have a crucial effect on the effective thickness of the determining part of the EDL it can be of importance to know the ionic 
composition inside the electrolyte. It also means that MFEM devices could be used to determine the approximate size of unknown charged particles.

\section{CONCLUSIONS}

We have shown and modeled ferroelectric polymer thin films polarized through an aqueous electrolyte and developed a simplified model for such a system which gives us a better idea of what the voltage, and charge distribution, as well as the electric field strength, in such a system looks like. This information provides us with insights into the conditions at electrolyte /ferroelectric polymer interface. The surface charge, and dynamics thereof, at this interface possibly provide us with a number of opportunities. Many chemical and biological systems are dependent on the surface energy and we here have an opportunity to control just that by controlling the polarization over the surface. The remnant switching of the ferroelectric only demands a short pulse after which the polarized surface can be disconnected from the circuit and easily placed in e.g. some closed chamber or incubator while keeping its properties. The different polarizations could possibly also attract differently charged particles to create patterns which will be maintained without consuming any additional power from the device. The relationship between the electric double layer thickness and the open circuit voltage could also provide a means to detect molecular changes at the surface. By monitoring the open circuit voltage, a chemical or biological coupling and its dynamics could most likely be detected if a probe was attached to the surface and an electrolyte containing the corresponding analyte were to flow over it.

\section{ACKNOWLEDGEMENTS}

The Authors would like to thank Xavier Crispin for theoretical input at the early stages of the project. This work supported by The Swedish Governmental Agency for Innovation Systems (VINNOVA, 201000507), The Knut and Alice Wallenberg Foundation, The Advanced Functional Materials Center at Linköping University and The Önnesjö Foundation.

\section{REFERENCES}

[1] Q. M. Zhang, V. Bharti, and X. Zhao, Science 280, 2101 (1998).

[2] T.-B. Xu, Z.-Y. Cheng, and Q. M. Zhang, Applied Physics Letters 80, 1082 (2002).

[3] R. C. G. Naber, C. Tanase, P. W. M. Blom, G. H. Gelinck, A. W. Marsman, F. J. Touwslager, S. Setayesh, and D. M. de Leeuw, Nat Mater 4, 243 (2005).

[4] S. Bauer, Journal of Applied Physics 80, 5531 (1996).

[5] S. J. Kang, Y. J. Park, I. Bae, K. J. Kim, H.-C. Kim, S. Bauer, E. L. Thomas, and C. Park, Advanced Functional Materials 19, 2812 (2009).

[6] S. B. Lang and S. Muensit, Appl. Phys. A 85, 125 (2006).

[7] S. Fabiano, X. Crispin, and M. Berggren, ACS Applied Materials \& Interfaces 6, 438 (2013).

[8] M. Dawber, P. Chandra, P. Littlewood, and J. Scott, Journal of Physics: Condensed Matter 15, L393 (2003).

[9] S. Jesse et al., Nat Mater 7, 209 (2008).

[10] S. V. Kalinin and D. A. Bonnell, Physical Review B 63, 125411 (2001).

[11] S. H. Lim, A. C. Rastogi, and S. B. Desu, Journal of Applied Physics 96, 5673 (2004).

[12] T. Lü and W. Cao, Physical Review B 66, 024102 (2002).

[13] N. Setter et al., Journal of Applied Physics 100 (2006). 
[14] D. A. Bernards, D. J. Macaya, M. Nikolou, J. A. Defranco, S. Takamatsu, and G. G. Malliaras, Journal of Materials Chemistry 18, 116 (2008).

[15] O. Teschke, G. Ceotto, and E. F. de Souza, Physical Review E 64, 011605 (2001).

[16] P. Thomas, R. S. E. Ravindran, and K. B. R. Varma, in Properties and Applications of Dielectric Materials (ICPADM), 2012 IEEE 10th International Conference on the2012), pp. 1.

[17] G. H. Gelinck, A. W. Marsman, F. J. Touwslager, S. Setayesh, D. M. de Leeuw, R. C. G. Naber, and P. W. M. Blom, Applied Physics Letters 87 (2005).

[18] H.-S. Xu, Z. Y. Cheng, V. Bharti, S. Wang, and Q. M. Zhang, Journal of Applied Polymer Science 75, 945 (2000).

[19] K. M. Persson, R. Karlsson, K. Svennersten, S. Löffler, E. W. H. Jager, A. RichterDahlfors, P. Konradsson, and M. Berggren, Advanced Materials 23, 4403 (2011).

[20] L. Faxalv, M. H. Bolin, E. W. H. Jager, T. L. Lindahl, and M. Berggren, Lab on a Chip 14, 3043 (2014).

[21] R. Ferris, B. Yellen, and S. Zauscher, Small 8, 28 (2012). 